Original Research Paper

\title{
Taxifolin Potently Diminishes Levels of Reactive Oxygen Species in Living Cells Possibly by Scavenging Peroxyl Radicals
}

\author{
Satoru Sakuma, Yui Kishiwaki, Mayu Matsumura, Hiroyuki Sawada, \\ Riko Hashimoto, Kohtaroh Gotoh, Kohhei Umemoto and Yohko Fujimoto \\ Department of Physiological Chemistry, \\ Osaka University of Pharmaceutical Sciences, 4-20-1 Nasahara, Takatsuki, Osaka 569-1094, Japan
}

\author{
Article history \\ Received: $19-05-2018$ \\ Revised: $23-05-2018$ \\ Accepted: 17-10-2018 \\ Corresponding Author: \\ Yohko Fujimoto \\ Department of Physiological \\ Chemistry, Osaka University of \\ Pharmaceutical Sciences, 4-20- \\ 1 Nasahara, Takatsuki, Osaka \\ 569-1094, Japan \\ Tell: +81726901055 \\ Fax: +81726901005 \\ Email: fujimoto@gly.oups.ac.jp
}

\begin{abstract}
Taxifolin, a naturally occurring dihydroflavonol, has been reported to possess antioxidant activity, whereas there is little information regarding its antioxidant potency as a flavonoid. In the present study, the antioxidant efficacy of taxifolin in living cells was compared to that of catechin, a potent radical scavenger, by using flow cytometry with the redox-sensitive fluorescent dye, $2^{\prime}, 7^{\prime}$-dichlorodihydrofluorescein diacetate. Further, diminishing efficacy of taxifolin on peroxyl radicals was assessed by using an electron spin resonance spin-trapping technique. Taxifolin significantly reduced tert-butyl hydroperoxide-induced increases in Reactive Oxygen Species (ROS) levels in Caco-2 cells; the effect of taxifolin was stronger than that of catechin. Taxifolin also scavenged peroxyl (ROO·) radicals with statistically significance compared to that of catechin. This study showed that taxifolin has the potential to diminish ROS levels in living cells, possibly by scavenging ROO.
\end{abstract}

Keywords: Taxifolin, Catechin, Peroxyl Radical, Antioxidant, Oxidative Stress

\section{Introduction}

Excessive amounts of Reactive Oxygen Species (ROS) contribute to the aging process and the development of various diseases such as atherosclerosis, diabetes, cancer, neurodegenerative diseases and liver cirrhosis (Basaga, 1990). To prevent the damages caused by ROS, organisms have developed an antioxidant defense system that includes non-enzymatic antioxidants and enzymes such as superoxide dismutase, catalase and glutathione peroxidase (Sorg, 2004). A second level of prevention against ROS-induced damage is constituted by other scavenging compounds present in the diet, especially from medicinal plants (Valko et al., 2007; Xia et al., 2010; Jorge et al., 2016).

From the perspective of free radical biology, plants encounter serious oxidative stress from strong UV-Vis light, atmospheric ROS, temperature changes and the processes of oxygen consumption for photosynthesis. Flavonoids are a main class of phenolic compounds and secondary plant metabolites generally located in leaves as water soluble glycosides in the vacuoles of plant cells (Bimpilas et al., 2015). Flavonoids are not only present in plants as constitutive agents but they also accumulate in plant tissues in response to microbial attack (Harborne and Williams, 2000).

Taxifolin is a dihydroflavonol that is abundant in citrus fruits and onions. Taxifolin is arguably the most interesting and promising component of dietary supplements or antioxidant-rich functional foods in the last two decades (Rice Evans et al., 1996; Topal et al., 2016; Li et al., 2017). Importantly, taxifolin exerts significant antioxidant effects that are critical in preventing the onset of apoptosis (Vladimirov et al., 2009). Moreover, taxifolin has been found to inhibit oxidative enzymes and the overproduction of ROS, thus ameliorating cerebral ischemia-reperfusion injury (Voulgari et al., 2010).

Until now, there has been little information concerning the antioxidant potency of taxifolin as a flavonoid. Therefore, in the present study, the efficacy of taxifolin to reduce ROS levels in living cells was compared with that of catechin, which is a well-known, potent radical scavenger (Harborne and Williams, 2000; 
Bimpilas et al., 2015), by using flow cytometry with the oxidation-sensitive fluorescent dye 2',7'dichlorodihydrofluorescein diacetate (DCFH-DA). The ability of taxifolin to scavenge peroxyl radicals (ROO·) by use of an Electron Spin Resonance (ESR) spin-trapping technique were also compared with that of catechin.

\section{Materials and Methods}

\section{Materials}

$(+)$ Taxifolin, $(+)$ catechin, chrysin, 6-hydroxy-2,5,7,8tetramethylchromane-2-carboxylic acid (Trolox) and tert-butyl hydroperoxide (t-BOOH, 70\%, w/w, aqueous solution) were obtained from Sigma Chemical Co., (St. Louis, MO, USA). DCFH-DA was obtained from Life Technologies Corporation (Carlsbad, CA, USA). $\alpha-(4-$ Pyridyl 1-oxide)-N-tert-butylnitrone (POBN) was purchased from Enzo Life Technologies (Farmingdale, NY, USA). $N$-Acetyl- $L$-Cysteine (NAC) and $\mathrm{Ce}\left(\mathrm{SO}_{4}\right)_{2} \cdot 4 \mathrm{H}_{2} \mathrm{O}$ were obtained from Wako Pure Chemical Industries, Limited (Osaka, Japan). All other reagents were analytical grade. The ultrapure water used in the present study was prepared with a compact ultrapure water system (Merck Millipore, Milli- $Q^{\circledR}$ ).

\section{Measurement of ROS in Caco-2 Cells}

Measurements of ROS in Caco-2 cells were performed by the method previously reported (Kohda et al., 2016). Caco-2 cells were purchased from the European Collection of Cell Cultures (Salisbury, Wilts, UK) and cultured in Minimum Essential Medium (Life Technologies Corporation) supplemented with $10 \%$ fetal bovine serum (Nichirei Biosciences Inc., Tokyo, Japan) and $1 \%$ non-essential amino acids (Life Technologies Corporation). The cells were maintained in a humidified atmosphere of $5 \%$ carbon dioxide at $37^{\circ} \mathrm{C}$.

The measurement of ROS was performed by flow cytometry with DCFH-DA. The cells $\left(1.0 \times 10^{6}\right.$ cells $/ 28$ $\mathrm{cm}^{2}$ dish) were incubated with the test reagents for $6 \mathrm{~h}$ and then DCFH-DA was added at a final concentration of $10 \mu \mathrm{M}$. After incubating for $30 \mathrm{~min}$, the cells were collected by centrifugation $\left(4^{\circ} \mathrm{C}\right.$ and $200 \times g$ for $\left.5 \mathrm{~min}\right)$ and washed twice. The samples were filtered through a nylon mesh $(37 \mu \mathrm{m})$ and subjected to flow cytometry (FACSAria $^{\mathrm{TM}}$ III flow cytometer, Becton Dickinson, Basel, Switzerland).

\section{ESR Measurement}

An ESR spectrometer, TE-2100 (JEOL, Tokyo, Japan) and a JEOL flat quartz cell were used. The conditions were: field, $336 \pm 5 \mathrm{mT}$; power, $4 \mathrm{~mW}$; field modulation, $0.200 \mathrm{mT}$; time constant, 0.1 ; and amplitude, 300. A manganese signal was used for the external standard.

\section{Observation of POBN-Signal Adducts Reflecting t-} BOO.

The $\mathrm{Ce}^{4+} / \mathrm{t}-\mathrm{BOOH}$ reaction was started by adding t$\mathrm{BOOH}$ (final concentration, $0.4 \mathrm{M}$ ) to a mixture of POBN (final concentration, $10 \mathrm{mM}$ ) and $\mathrm{Ce}\left(\mathrm{SO}_{4}\right)_{2} \cdot 4 \mathrm{H}_{2} \mathrm{O}$ (final concentration, $0.2 \mathrm{mM}$ ) in $0.1 \mathrm{M}$ sodium phosphate buffer ( $\mathrm{pH} 7.4$ ) in a total volume of $0.5 \mathrm{~mL}$. The POBN-signal adducts reflecting t-BOO- were measured $1 \mathrm{~min}$ after the addition of $\mathrm{t}-\mathrm{BOOH}$.

\section{Statistical Analysis}

The results are expressed as means \pm standard errors of the mean. Significant differences between two groups were assessed using $t$-tests, whereas differences between multiple groups were assessed by one-way analysis of variance, followed by Scheffé's multiple comparison tests. $P$-values less than 0.05 were considered statistically significant.

\section{Results}

Taxifolin Suppresses ROS Generation Induced by $t$ BOOH in Caco-2 Cells

Figure 1A shows the effects of $\mathrm{t}-\mathrm{BOOH}$ with or without taxifolin on the intracellular ROS generation of Caco-2 cells, measured by flow cytometry with the redox-sensitive fluorescent dye, DCFH-DA. The addition of t-BOOH (50 $\mu \mathrm{M})$ to the Caco-2 cells shifted the Mean Fluorescence Intensity (MFI, dashed line) to the right, which indicates an increase in ROS levels measured by the DCF fluorescence. The increment in MFI induced by $\mathrm{t}-\mathrm{BOOH}$ was reduced by the addition of $50 \mu \mathrm{M}$ taxifolin. Figure $2 \mathrm{~B}$ summarizes the MFI data measured by the method in Fig. 1A. The addition of NAC, which is often used as an antioxidant in cell experiments (Lasram et al., 2015), diminished the tBOOH-induced increase in MFI ( $2 \mathrm{mM}, 50 \%$ inhibition). Fifty micromolar of both taxifolin (61\% inhibition) and catechin (39\% inhibition), but not chrysin, reduced the t$\mathrm{BOOH}$-induced increase in MFI. The taxifolin effect was stronger than that of catechin and their difference was statistically significant. This means that taxifolin reduces the t-BOOH-induced increase in ROS levels in living cells stronger than catechin does.

\section{Taxifolin Quenches $t-B O O$.}

A direct method for measuring free radicals in aqueous conditions is detection by ESR spectroscopy (Venkataraman et al., 2004). Figure 2A shows the ESR spectra of the spin signal adduct from t-BOO $[\mathrm{a}(\mathrm{N})=$ $1.51 \mathrm{mT}, \mathrm{a}(\mathrm{H})=0.23 \mathrm{mT}]$ by the reaction of the $\mathrm{Ce}^{4+} / \mathrm{t}-$ $\mathrm{BOOH}$ system with POBN. The hyperfine fit parameters are identical to those previously reported (Panasenko et al., 2002 ; 2005). An obvious quenching by $0.5 \mathrm{mM}$ Trolox, which is known as a potent peroxyl radical scavenger 
(Barclay et al., 1995; Stefek et al., 2005), supported the identity of the product. A positive correlation between the disappearance of the signal intensity of POBN signal adducts of t-BOO and trolox concentrations was observed (Supplementary Fig. 1). Figure 2A and Supplementary Fig. 1 collectively demonstrate that this experimental condition using the ESR apparatus can assess the diminishing efficacy of specific substances against t-BOO.
Figure 2B shows the effects of taxifolin, catechin and chrysin on the spin signal adduct of t-BOO- detected by the methods used in Fig. 2A. Both Taxifolin and catechin, but not chrysin, from 5 to $200 \mu \mathrm{M}$ concentration-dependently diminished the signal adduct of t-BOO· [taxifolin, 34-90\% inhibition; catechin, 3-82\% inhibition). The scavenging effect of $200 \mu \mathrm{M}$ taxifolin was stronger than that of $200 \mu \mathrm{M}$ catechin and the difference was statistically significant.

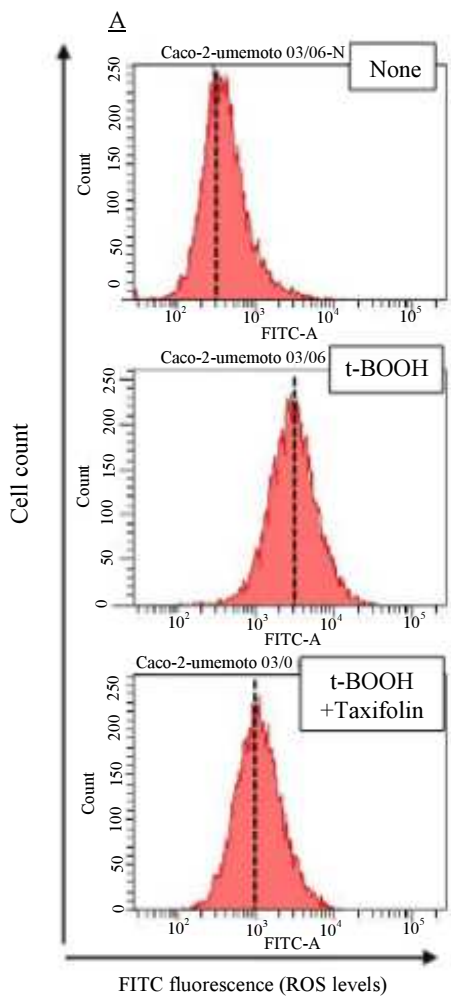

$\underline{B}$

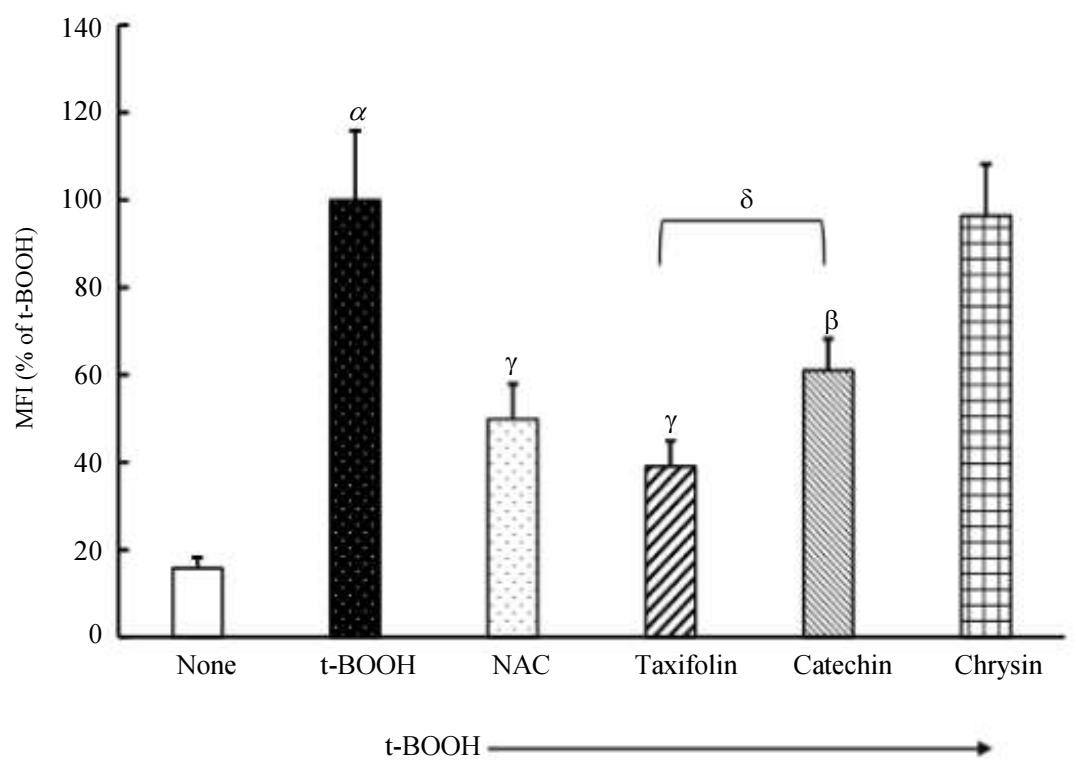

Fig. 1: Alterations in ROS levels in Caco-2 cells treated with t-BOOH in combination with taxifolin, catechin and chrysin; (A) Representative flow cytometry univariate histograms. B) Quantitative assessment of the MFI flow cytometry data. The data are presented as means \pm standard errors of the mean $(\mathrm{n}=7) .{ }^{\alpha} p<0.01$ vs. None. ${ }^{\beta} p<0.05,{ }^{\gamma} p<0.01 \mathrm{Vs}$. t-BOOH. ${ }^{\delta} p<0.05$ vs. catechin. MFI, mean fluorescence intensity (shown as dashed line in part A); t-BOOH, tert-butyl hydroperoxide; NAC, $N$ acetyl- $L$-cysteine.t-BOOH, $50 \mu \mathrm{M}$; NAC, $2000 \mu \mathrm{M}$; taxifolin, $50 \mu \mathrm{M}$; catechin, $50 \mu \mathrm{M}$; chrysin, $50 \mu \mathrm{M}$

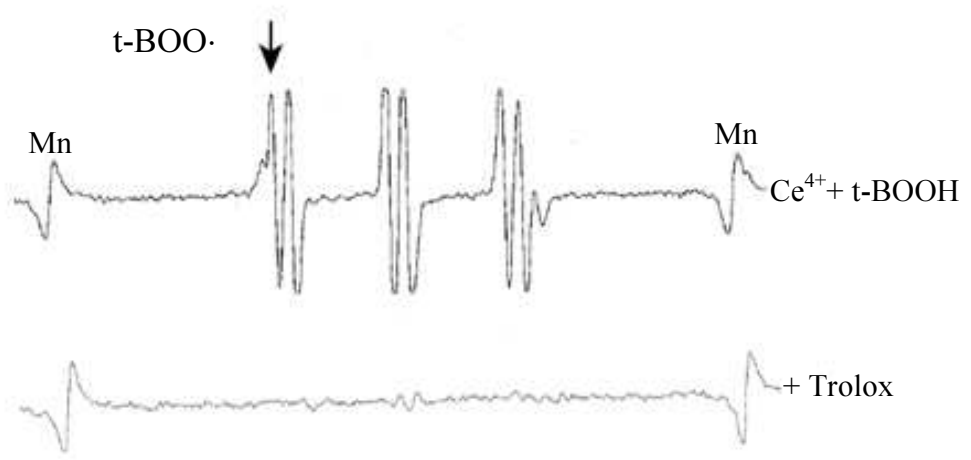




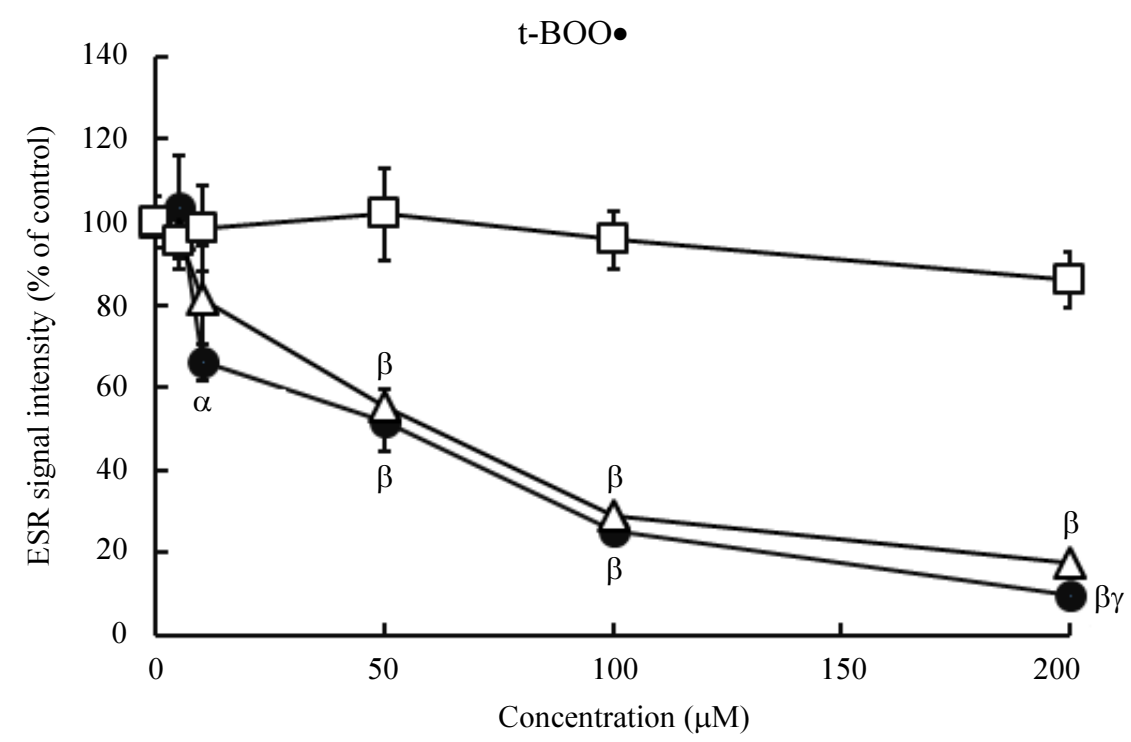

(B)

Fig. 2: Effects of taxifolin, catechin and chrysin on the amounts of spin signal adducts of t-BOO• generated by the chemical reaction system; (A) Representative ESR signal spectra of POBN spin adduct reflecting t-BOO•. Ce $\mathrm{e}^{4+}, 0.2 \mathrm{mM}$; $\mathrm{t}-\mathrm{BOOH}, 400 \mathrm{mM}$; Trolox, $0.5 \mathrm{mM}$. (B) Effects of taxifolin on ESR signal spectra. The radical intensity was defined as the ratio of the peak height of signal (indicated as an arrow in part A) to that of Manganese (Mn). The data are presented as means \pm standard errors of the mean $(\mathrm{n}=3)$. ${ }^{\alpha} p<0.05,{ }^{\beta} p<0.01$ vs. control. ${ }^{\gamma} p<0.05$ vs. $200 \mu \mathrm{M}$ catechin. t-BOO•, tert-butyl peroxyl radical; ESR, electron spin resonance; t-BOOH, tert-butyl hydroperoxide

The results of Fig. 2 show that taxifolin diminishes t$\mathrm{BOO}$ and the t-BOO scavenging efficacy seems to be somewhat greater than that of catechin.

\section{Discussion}

ROS contributes to the development of various diseases such as atherosclerosis, diabetes, cancer, neurodegenerative diseases and liver cirrhosis. It also contributes to the aging process (Basaga, 1990). The use of antioxidant compounds, such as radical scavengers, might prevent the development and progression of these diseases to maintain health.

As shown in Fig. 1, taxifolin suppressed t-BOOHinduced increases in ROS levels in Caco-2 cells and the effect of $200 \mu \mathrm{M}$ taxifolin was significantly stronger than that of catechin. Based on an ESR technique, Fig. 2 showed that taxifolin could diminish ROO- and $200 \mu \mathrm{M}$ taxifolin's effect was significantly stronger than that of catechin. Chrysin was without effects on both ROS levels in Caco-2 cells and ESR radical intensity of the spin signal adduct of t-BOO. Both taxifolin and catechin have a catechol group in ring $\mathrm{B}$ and taxifolin possesses an additional 4-oxo group in ring $\mathrm{C}$ (Supplementary Fig. 2). Thus, it is possible that the catechol group in ring B plays an important role for the ROO- scavenging activity and the 4-oxo group in ring $\mathrm{C}$ determines the strength of the ROO- scavenging capacity of taxifolin. The belief that the catechol group in ring $\mathrm{B}$ of flavonoids is the major structural feature imparting antioxidant activity has been supported by the work of Jovanovic et al. (1996).

Topal et al. (2016) and $\mathrm{Li}$ et al. (2017) have reported that taxifolin is an effective antioxidant and antiradical by using indirect in vitro bioanalytical methods, including 1,1-diphenyl-2-picryl-hydrazyl radical-scavenging, 2,2'-azino-bis (3-ethylbenzothiazoline-6-sulfonic acid) radical-scavenging, $\mathrm{Fe}^{3+}$ reducing and $\mathrm{Cu}^{2+}$-reducing assays. However, to our knowledge, there is no information concerning the types of ROS scavenged by taxifolin by using an ESR method. In the present study, we showed that taxifolin scavenged ROO. Li et al. (2017) has reported that taxifolin is an effective scavenger of hydroxyl radical $(\cdot \mathrm{OH})$. The present study together with the report by $\mathrm{Li}$ et al. (2017) indicates that taxifolin can be a scavenger of both $\mathrm{ROO} \cdot$ and $\cdot \mathrm{OH}$.

Determining the significance of the present finding that taxifolin quenches t-BOO- will require further study. Additional studies are also needed to clarify the mechanism by which taxifolin scavenges ROO. However, to the best of our knowledge, the results of this study show for the first time that taxifolin diminishes ROS levels in living cells possibly by scavenging ROO· This provides new mechanistic insight into the preventive effects of taxifolin in various disorders. 


\section{Conclusion}

Taxifolin significantly reduced $\mathrm{t}$-BOOH-induced increases in ROS levels in Caco-2 cells and this effect was stronger than that of another flavonoid, catechin. Taxifolin also scavenged ROO by using an ESR method. We believe that our study makes a significant contribution to the literature because, to the best of our knowledge, this is the first time that taxifolin has the potential to diminish ROS levels in living cells possibly by scavenging ROO.

\section{Authors Contributions}

Satoru Sakuma: Participated in all experiments, coordinated the data-analysis and contributed to the writing of the manuscript.

Yui Kishiwaki, Mayu Matsumura, Hiroyuki Sawada, Riko Hashimoto, Kohtaroh Gotoh, Kohhei Umemoto and Yohko Fujimoto: Participated in some experiments, coordinated the data-analysis.

\section{Ethics}

This article is original and contains unpublished material. The corresponding author confirms that all of the other authors have read and approved the manuscript and there are no ethical issues involved.

\section{References}

Barclay, L.R.C., J.D. Artz and J.J. Mowat, 1995. Partitioning and antioxidant action of the watersoluble antioxidant, Trolox, between the aqueous and lipid phases of phosphatidylcholine membranes: ${ }^{14} \mathrm{C}$ tracer and product studies. Biochim. Biophys. Acta, 1237: 77-85. DOI: 10.1016/0005-2736(95)00071-A

Basaga, H.S., 1990. Biochemical aspects of free radicals. Biochem. Cell. Biol., 68: 989-998.

DOI: $10.1139 / 090-146$

Bimpilas, A., D. Tsimogiannis, K. Balta-Brouma, T. Lymperopoulou and V. Oreopoulou, 2015. Evolution of phenolic compounds and metal content of wine during alcoholic fermentation and storage. Food Chem., 178: 164-171.

DOI: 10.1016/j.foodchem.2015.01.090

Harborne, J.B. and C.A. Williams, 2000. Advances in flavonoid research since 1992. Phytochemistry, 55: 481-504.

Jorge, E.G., A.M. Rayar, S.J. Barigye, M.E.J. Rodríguez and M.S.I. Veitía et al., 2016. Development of an in silico model of DPPH. Free radical scavenging capacity: Prediction of antioxidant activity of coumarin type compounds. Int. J. Mol. Sci., 17: 881-891. DOI: $10.3390 /$ ijms 17060881
Jovanovic, S.V., S. Steenken, Y. Hara and M.G. Simic, 1996. Reduction potentials of flavonoids and model phenoxy radicals. Which ring in flavonoids is responsible for antioxidant activity? J. Chem. Soc. Perkin Trans., 2: 2497-2504. DOI: $10.1039 / \mathrm{P} 29960002497$

Kohda, T., S. Sakuma and Y. Fujimoto, 2016. Vitamin E-like molecules potentiate the curcumin-induced suppression of Caco-2 cell proliferation. Int. J. Pharmacol. Res., 6: 41-46.

Lasram, M.M., I.B. Dhouib, A. Annabi, S. El Fazaa and N. Gharbi, 2015. A review on the possible molecular mechanism of action of $\mathrm{N}$-acetylcysteine against insulin resistance and type-2 diabetes development. Clin. Biochem., 48: 1200-1208. DOI: 10.1016/j.clinbiochem.2015.04.017

Li, X., H. Xie, Q. Jiang, G. Wei and L. Lin et al., 2017. The mechanism of $(+)$ taxifolin's protective antioxidant effect for $\bullet \mathrm{OH}$-treated bone marrowderived mesenchymal stem cells. Cell. Mol. Biol. Lett., 22: 31-42. DOI: 10.1186/s1 1658-017-0066-9

Panasenko, O.M., A.N. Osipov, A.V. Chekanov, J. Arnhold and V.I. Sergienko, 2002. Peroxyl radical is produced upon the interaction of hypochlorite with tert-butyl hydroperoxide. Biochemistry (Moscow), 67: 1061-1070. DOI: 10.1023/A:1019962519941

Panasenko, O.M., A.V. Chekanov, J. Arnhold, V.I. Sergienko and A.N. Osipov et al., 2005. Generation of free radicals during decomposition of hydroperoxide in the presence of myeloperoxidase or activated neutrophils. Biochemistry (Moscow), 70: 998-1004. DOI: 10.1007/s10541-005-0215-z

Rice Evans, C.A., N.J. Miller and G. Paganga, 1996. Structure-antioxidant activity relationships of flavonoids and phenolic acids. Free Radic. Biol. Med., 20:933-56. DOI: $10.1016 / 0891-5849(95) 02227-9$

Sorg, O., 2004. Oxidative stress: A theoretical model or a biological reality? C. R. Biol., 327: 649-662. DOI: $10.1016 /$ j.crvi.2004.05.007

Stefek, M., Z. Kyselova, L. Rackova and L. Krizanova, 2005. Oxidative modification of rat eye lens proteins by peroxyl radicals in vitro: Protection by the chainbreaking antioxidants stobadine and Trolox. Biochim. Biophys. Acta, 1741: 183-190. DOI: $10.1016 /$ j.bbadis.2005.01.006

Topal, F., M. Nar, H. Gocer, P. Kalin and U.M. Kocyigit et al., 2016. Antioxdant activity of taxifolin: An activity-structure relathionship. J. Enzyme Inhib. Med. Chem., 31: 674-683. DOI: $10.3109 / 14756366.2015 .1057723$

Valko, M., D. Leibfritz, J. Moncol, M.T.D. Cronin and M. Mazur et al., 2007. Free radicals and antioxidants in normal physiological functions and human disease. Int. J. Biochem. Cell. Biol., 39: 44-84. DOI: 10.1016/j.biocel.2006.07.001 
Venkataraman, S., F.Q. Schafer and G.R. Buettner, 2004. Detection of lipid radicals using EPR. Antioxid. Redox Singal., 6: 631-638.

DOI: $10.1089 / 152308604773934396$

Vladimirov, Y.A., E.V. Proskurnina, E.M. Demin, N.S. Matveeva and O.B. Lubitskiy et al., 2009. Dihydroquercetin (taxifolin) and other flavonoids as inhibitors of free radical formation at key stages of apoptosis. Biochemistry (Mosc), 74: 301-307.

DOI: $10.1134 / \mathrm{S} 0006297909030092$
Voulgari, C., D. Papadogiannis and N. Tentolouris, 2010. Diabetic cardiomyopathy: From the pathophysiology of the cardiac myocytes to current diagnosis and management strategies. Vasc. Health Risk Manag., 6: 883-903.

DOI: $10.2147 /$ VHRM.S11681

Xia, E.Q., G.F. Deng, Y.J. Guo and H.B. Li, 2010. Biological activities of polyphenols from grapes. Int. J. Mol. Sci., 11: 622-646.

DOI: $10.3390 / \mathrm{ijms} 11020622$

\section{Supplementary Material}

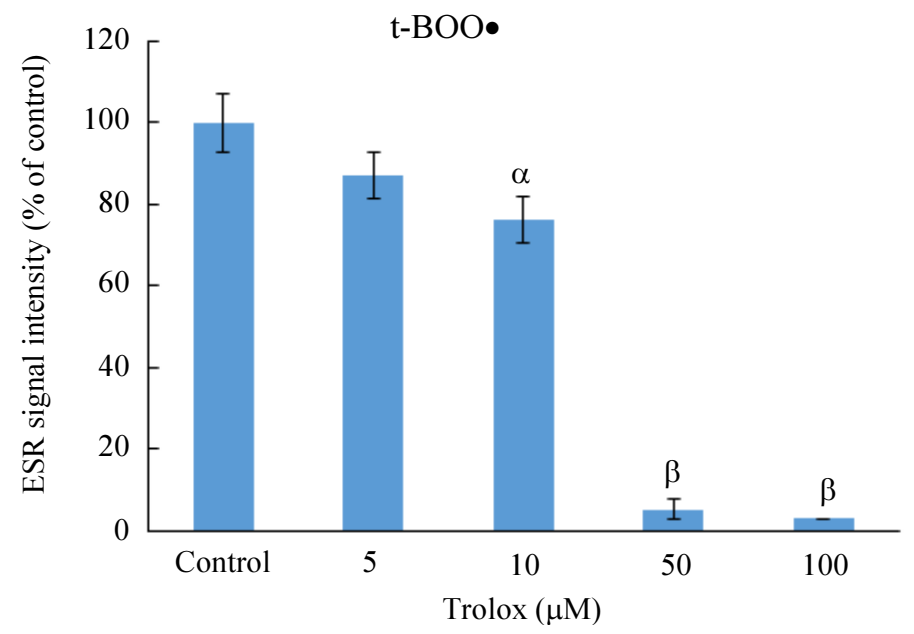

Supplementary Fig. 1: Effects of trolox on the amounts of spin signal adduct of t-BOO• generated by the chemical reaction system

The radical intensity was defined as the ratio of the peak height of signal [indicated as an arrow in Fig. 2(A)] to that of Manganese (Mn). The data are presented as means \pm standard errors of the mean $(\mathrm{n}=3) .{ }^{\alpha} p<0.05,{ }^{\beta} p<0.01$ vs. Control. Electron-spin resonance (ESR) coupled with spin-trapping technique used in this study is shown in Material and Methods and Results and Discussion sections in the manuscript.<smiles>O=C1c2c(O)cc(O)cc2O[C@H](c2ccc(O)c(O)c2)[C@@H]1O</smiles>

Taxifolin<smiles>Oc1cc(O)c2c(c1)O[C@H](c1ccc(O)c(O)c1)C(O)C2</smiles>

Catechin<smiles>O=c1cc(-c2ccccc2)oc2cc(O)cc(O)c12</smiles>

Supplementary Fig. 2: Structures of taxifolin, catechin and chrysin 